K. GANESAN

\title{
Trends in solar power plant automation technologies
}

As solar power continues to grow, plant automation and control becomes more important. It comprises all the necessary intelligence to turn the solar energy into a reliable and flexible power source. As leading automation provider, field proven PLC (Programmable Logic Controllers) and SCADA (Supervisory Control and Data Acquisition) system accompanied by dedicated control and diagnostics functions assure reliable power production and grid code compliance at all times.

\subsection{Introduction}

$\mathrm{S}$ olar power plant automation comprises measuring sensors, instruments, controllers, actuators, networks, industrial computers, and interfaces that manage the control of solar power plants and automated grid interfacing. Mainly PLCs (Programmable Logic Controllers) allow inter connection of those systems through data collection. They are used in all types of industries; from electrical distribution systems, to food processing, to power plants. Traditionally, SCADA systems have made use of the public switched network (PSN) for monitoring purposes to save time. Today many systems are monitored using the infrastructure of the corporate local area network (LAN)/wide area network (WAN) and industrial networking. Another common term used to describe a SCADA system is just like to human machine interface (HMI). It is used to describe any system that provides an interface between a person and a piece of machinery in a field. The industrial SCADA system falls into this category for monitoring. It provides an HMI by displaying process variables to the operator and allowing control of the plant

\subsection{Functions of SCADA}

The first of which is to display information about the current operating conditions of a plant in an informative and graphical interface/trends and the second is to allow supervisory control of the plant by personnel/data acquisition. Larger commercial systems may also have other features, such as historical trending/graphically of data to

Blind peer reviews carried out

Mr. K. Ganesan, ADGM, C\&I, Thermal Power Station-II, NLC India Limited, E-mail: K.ganesan@nlcindia.com allow the past operation of the plant to be recorded for future reference and for faultfinding. These other features are secondary to the main purpose of the SCADA [1].

\subsection{Components of SCADA System}

These are the field data interface devices (IEDs), remote terminal units (RTUs), communications medium, master station (central host computer) and operator workstations. Field data interface devices (IEDs): Digital or analogue intelligent electronic devices and control relays that directly interface with the managed system.

\subsubsection{Light sensor}

Light sensor generates an output signal indicating the intensity of light by measuring the radiant energy that exists in a very narrow range of frequencies basically called "light", and which ranges in frequency from "infrared" to "visible" up to "ultraviolet" light spectrum. A light sensor is a passive or active devices that convert this "light energy" whether visible or in the infrared parts of the spectrum into an electrical signal output. Light sensors are more commonly known as "photoelectric devices" or "photo sensors" because the convert light energy (photons) into electricity (electrons).

\subsubsection{Temperature sensor (LM35)}

New types of precision integrated-circuit temperature sensors, whose output voltage is linearly proportional to the Celsius (Centigrade) temperature, are generally used.

\subsubsection{Humidity sensor}

Humidity sensors are gaining more significance in diverse areas of measurement and control technology. Manufacturers are not only improving the accuracy and long term drift of their sensors, they are improving their durability for use in different environments, and simultaneously reducing the component size and the price.

\subsubsection{Hall effect sensor}

A hall effect sensor is a transducer that varies its output voltage in response to a magnetic field. Hall effect sensors are used for proximity switching, positioning, speed detection, and current sensing applications.

\subsubsection{Voltage sensor}

In electronics, a voltage divider (also known as a potential 


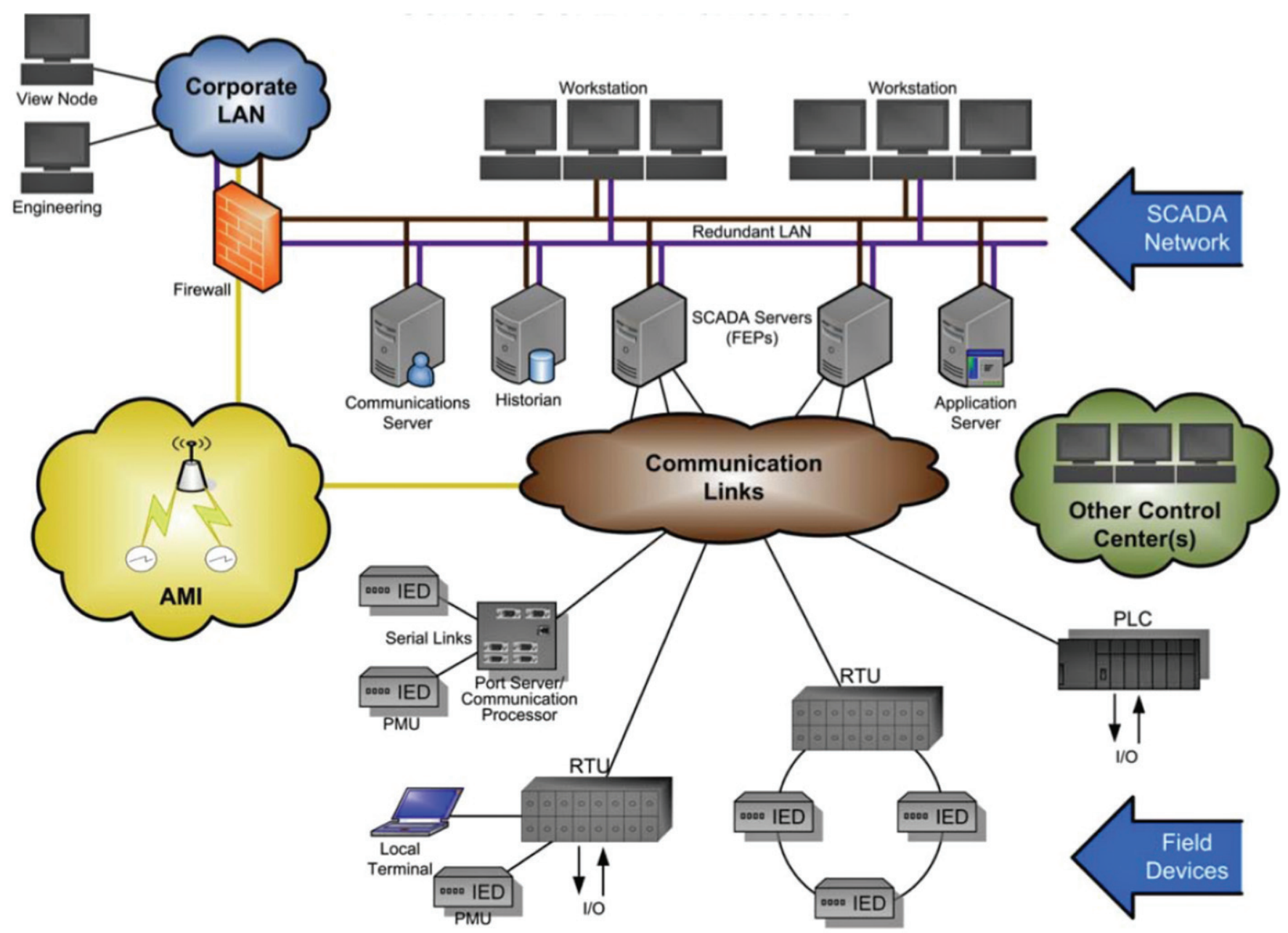

Fig.1 Components of SCADA

divider) is a simple linear circuit that produces an output voltage (Vout) that is a fraction of its input voltage (Vin). Voltage division refers to the partitioning of a voltage among the components of the divider.

A simple example of a voltage divider consists of two resistors in series or a potentiometer. It is commonly used to create a reference voltage, and may also be used as a signal.

\subsubsection{Current sensor}

Remote terminal unit (RTUs): Gathers information from their remote site from various intelligent electronic devices. They are primarily used to convert electronic signals received from field interface devices into the language used to transmit the data over a communication channel.

Communications medium: The devices used to connect the SCADA master unit to the RTUs in the field.

The master station: Initiates all communication, gathers data, stores information, sends information to other systems, and interfaces with operators. The major difference between the master station and RTU is that the master station initiates virtually all communication between the two.
Operator workstations: Operator workstations are most often computer terminals that are networked with the SCADA central host computer [4].

\subsection{Software components}

SCADA software is divided into two types; proprietary or open. Companies develop proprietary software to communicate to their hardware. The main problem with these systems is the overwhelming reliance on the supplier of the system. Open software systems have gained popularity because of the interoperability they bring to the system.

Key features of SCADA software include user interfaces, graphics displays, alarms, trends, RTU (and PLC) interface, scalability, access to data, database, networking, fault tolerance and redundancy, and client/server distributed processing.

\subsection{Software FEATURe SCADA}

- Log in page for security

- Configuration

- SCADA status and geographical map 
- Real time trend

- Historical graph

- Alarm and event logging

- Inverter status and alarm

- AC, DC voltage and current data logging

- Electricity production logging

- Temperature, wind, Pyrano meter logging

- Report for electricity production in hourly, daily, monthly

\subsection{SCADA COMMUNICATION PROTOCOLS}

SCADA Communications protocols define the method by which data is transmitted along a communication link. The data representations in a SCADA network are identified not in any fashion other than by unique addressing. The addressing is designed to correlate with the SCADA master station database. Each protocol consists of two message sets. One set forms the master protocol, containing the valid statements for master station initiation or response, and the other set is the RTU protocol, containing the valid statements an RTU can initiate and respond to. In most of the cases, these pairs can be considered a poll or request for information or action and a confirming response.

\subsection{SCADA SYSTEM}

The components of the SCADA system combine to provide auto-remote control and switching capabilities into GRID Co network and ECG's Primary network. The system provides the operators monitoring the HMI with sufficient information to operate the network. Furthermore, it gives warning and alarms for unexpected or critical events; allow remote control and post fault analysis.

The section of the power system that has benefited from SCADA is from the generation section through to the high voltage transmission network and sub-transmission network of the distribution utilities. There is however no remote means of monitoring the condition of power transformers in the distribution utility companies which is a vital component of the power system.

\subsection{Programmable logic controller components}

A programmable controller is a solid state user programmable control system with functions to control logic, sequencing, timing, arithmetic data manipulation and counting capabilities. It can be viewed as an industrial computer that has a central processor unit, memory, input output interface and a programming device. The central processing unit provides the intelligence of the controller. It accepts data, status information from various sensing devices like limit switches, proximity switches, executes the user control programme stored in the memory and gives appropriate output commands to devices such as solenoid valves, switches etc.

Input output interface is the communication link between field devices and the controllers. Through these interfaces the processor can sense and measure physical quantities regarding a machine or process, such as, proximity, position, motion, level, temperature, pressure, etc. Based on status sensed, the CPU issues command to output devices such as valves, motors, alarms, etc. The programmer unit provides the man machine interface. It is used to enter the application programme, which often uses a simple user-friendly logic.

\subsection{COMPONENTS}

The PLC mainly consists of a CPU, memory areas, and appropriate circuits to receive input/output data.

We can actually consider the PLC to be a box full of hundreds or thousands of separate relays, counters, timers and data storage locations. They do not physically exist but rather they are simulated and can be considered software counters, timers, etc. Each component of a PLC has a specific function:

Input relays (contacts) - These are connected to the outside world. They physically exist and receive signals from switches, sensors, etc. Typically they are not relays but rather they are transistors.

Internal utility relays - These do not receive signals from the outside world nor do they physically exist. They are simulated relays and are what enables a PLC to eliminate external relays. There are also some special relays that are dedicated to performing only one task. Some are always on while some are always off. Some are on only once during power-on and are typically used for initializing data that was stored.

Counters - These are simulated counters and they can be programmed to count pulses. Typically these counters can count up, down or both up and down since they are simulated they are limited in their counting speed. Some manufacturers also include high-speed counters that are hardware based. We can think of these as physically existing.

Timers - These come in many varieties and increments. The most common type is an on-delay type. Others include off-delay and both retentive and non-retentive types. Increments vary from 1 millisecond through 1 second.

Output relays (coils) - These are connected to the outside world. They physically exist and send on/off signals to solenoids, lights, etc. They can be transistors, relays, or tracs depending upon the model chosen.

Data storage - Typically there are registers assigned to simply store data. They are usually used as temporary storage for math or data manipulation.

They can also typically be used to store data when power is removed from the PLC. Upon power-up they will still have the same contents as before power was removed.

The block diagram of Plant Level Monitoring with tracker (PLM-2) having the same components as of PLM-1 with additional solar tracker. The solar panel tracks sun according 


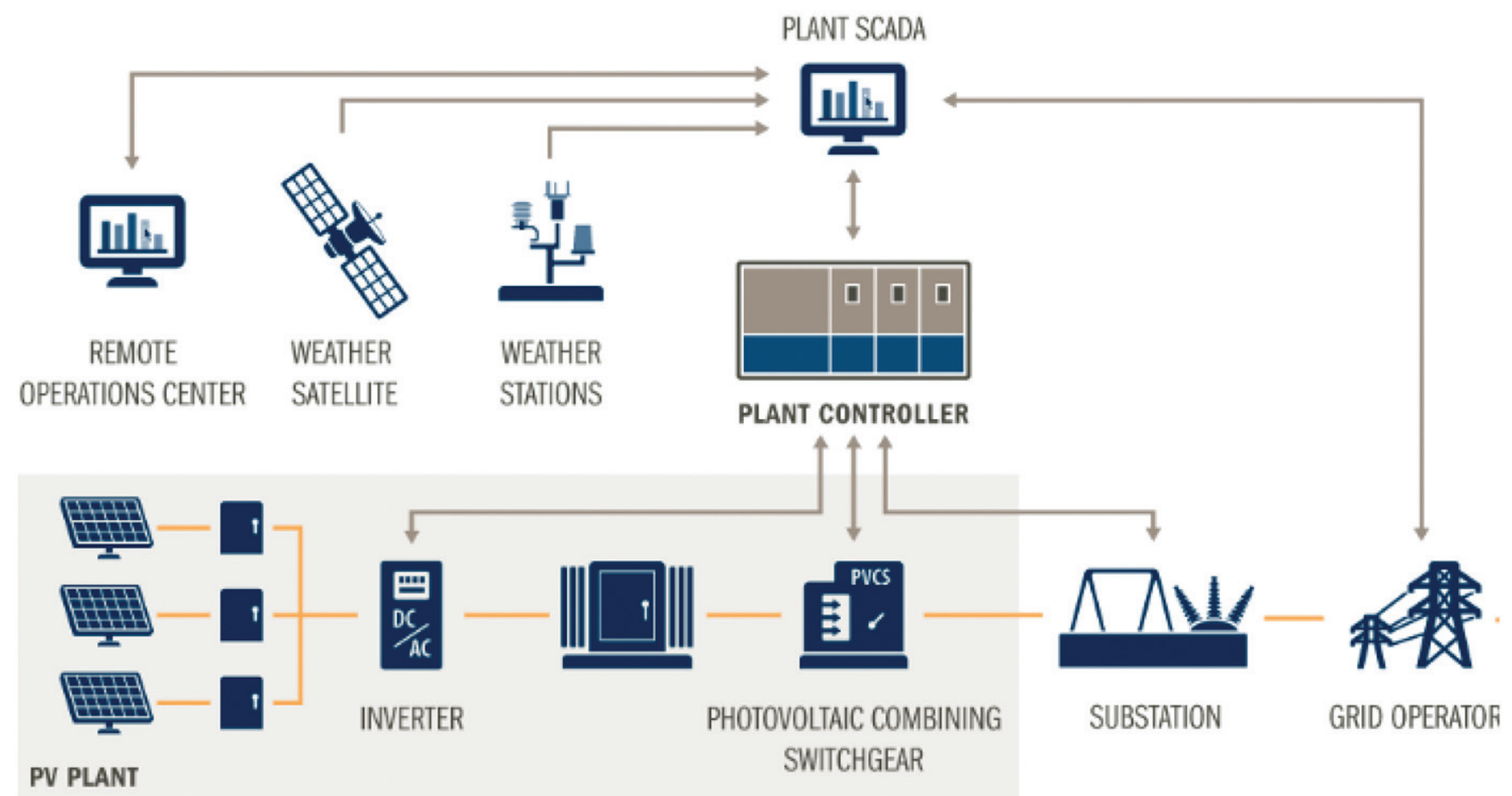

Fig.2 basic block diagram industrial networking system

to the time by using RTC peripheral. In this system we are calculating the amount of power generated by PLM-1, PLM2 , and compare this to know which plant is more efficient. If any of solar panel is not working we can know directly by the values, which are updated from the plant to central station for every minute. In PLM we can prevent the short circuit between solar panel and inverter by DC breaker circuits. As the values of the plant are updated to central station we can continuously monitor the plant from central station. Central control station consists of wireless module, alert system, PC GUI, grid sensing unit, AC voltage sensor, AC current sensor and inverter. Central control station will process the data from PLM's and the availability of the line grip power it will start the inverter functionality. Load monitoring units measure the values of AC current, AC voltage and AC power which map to the grid. All these information will send to the monitoring for analysis purpose.

The sensors data from the plant are updated to the central control station through wireless module and displayed in the GUI. The amount of power generated by the plant is calculated and displayed in GUI. Whenever grid wants the power supply, firstly it checks the status of the grid. If grid is available then central station on the inverter so that the solar panel generated power is fed to inverter which converts DC power to $\mathrm{AC}$ power. The amount of power supplied to the grid can be calculated by $\mathrm{AC}$ current sensor and $\mathrm{AC}$ voltage sensor. If the power supply required for the grid is not available then alert system i.e. buzzer is on and off the inverter functionality.
A typical real time controller will connect to remote $\mathrm{I} / \mathrm{O}$ to collect the data from inverter and current PV from the combiner box and also included is the alarm of surge detection in the box. The real time controller will interface to 5 remote I/O equipment system which support 32 analog inputs and 32 digital inputs.

The core network boasts fiber optic cabling with star topology for highly efficient capability and for protection from the power surge from lightning to communication line (wireless network options). The controller performs as a data logging and control system to collect all data from inverter, meter, temperature sensor, wind sensor and pyrano meter and the current of solar combiner box.

An industrial computer functions as a status management and also handles remote monitoring tasks from remote areas to manage all equipment at site. Also, the computer stores the backup data from the real time controller whenever the transmission is lost and sends the backup data to data center when the transmission resumes back. The controller will send all data to server via GPRS modem and store all of the data into memory. In case the network GPRS is down, the controller will store the backup data and will send the backup data to the server whenever the network is recovered. The user can use an internet browser to monitor all the meters and status of the equipment which include historical, real time data, graph and alarm logging. The client software can connect to web browser to login to the system and to see the SCADA real time status, alarm, event, power consumption, communication efficiency, inverter status, electricity 
production, $\mathrm{DC}$ and $\mathrm{AC}$ voltage and current logging and trend graph etc.

\subsection{Plant LeVel Control}

A software library in the control system coordinates the plant's active and reactive power production to comply with the grid codes:

- Central plant controller coordinates all inverters to achieve the required control command

- Limits power production (active and reactive) of the plant according to requested set point

- Controls the ramp rate of power production according to specifications in grid codes

- Provides power factor, voltage and frequency control at the point of connection to the utility grid
- Accounts for outages and scheduled maintenance of the inverters

The intermittent behaviour of the PV generator is supervised by the power electronic converter processing the generated power of the PV generator. Through a feature known as maximum power point tracking (MPPT), the converter monitors the output power of the generator, adjusting it constantly to the desired level by changing the voltage level of the generator.

Another interesting development emerging in PV applications for larger systems is the inclusion of environmental measurement data to enhance near-future prediction and power output. Through monitoring, e.g., the cloud movement near the PV power plant, the centralized controller can, in advance, guide the inverter(s) within the
Substation

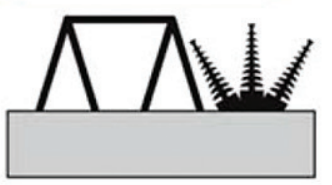

Substation remote terminal unit

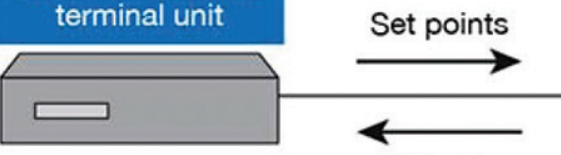

RTU data
Point of interconnection measurements
SCADA humanmachine interface

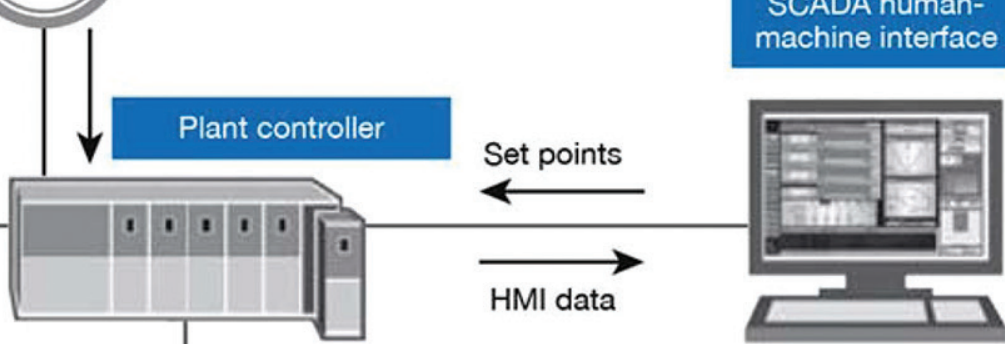

Set points (power, voltage or PF)

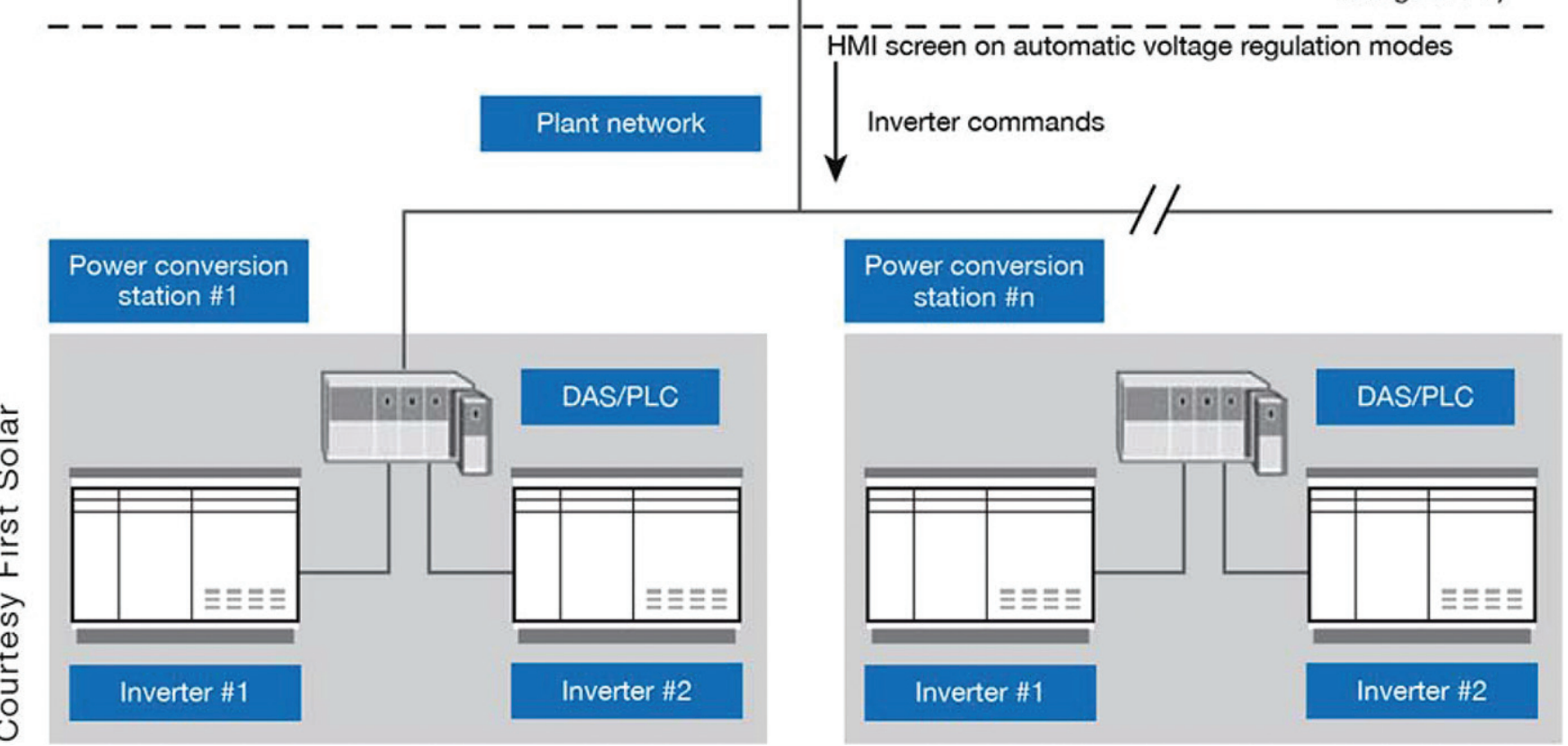

Fig.3 Utility-scale PV plants - illustrated the basic architecture and components of a plant-level control system 


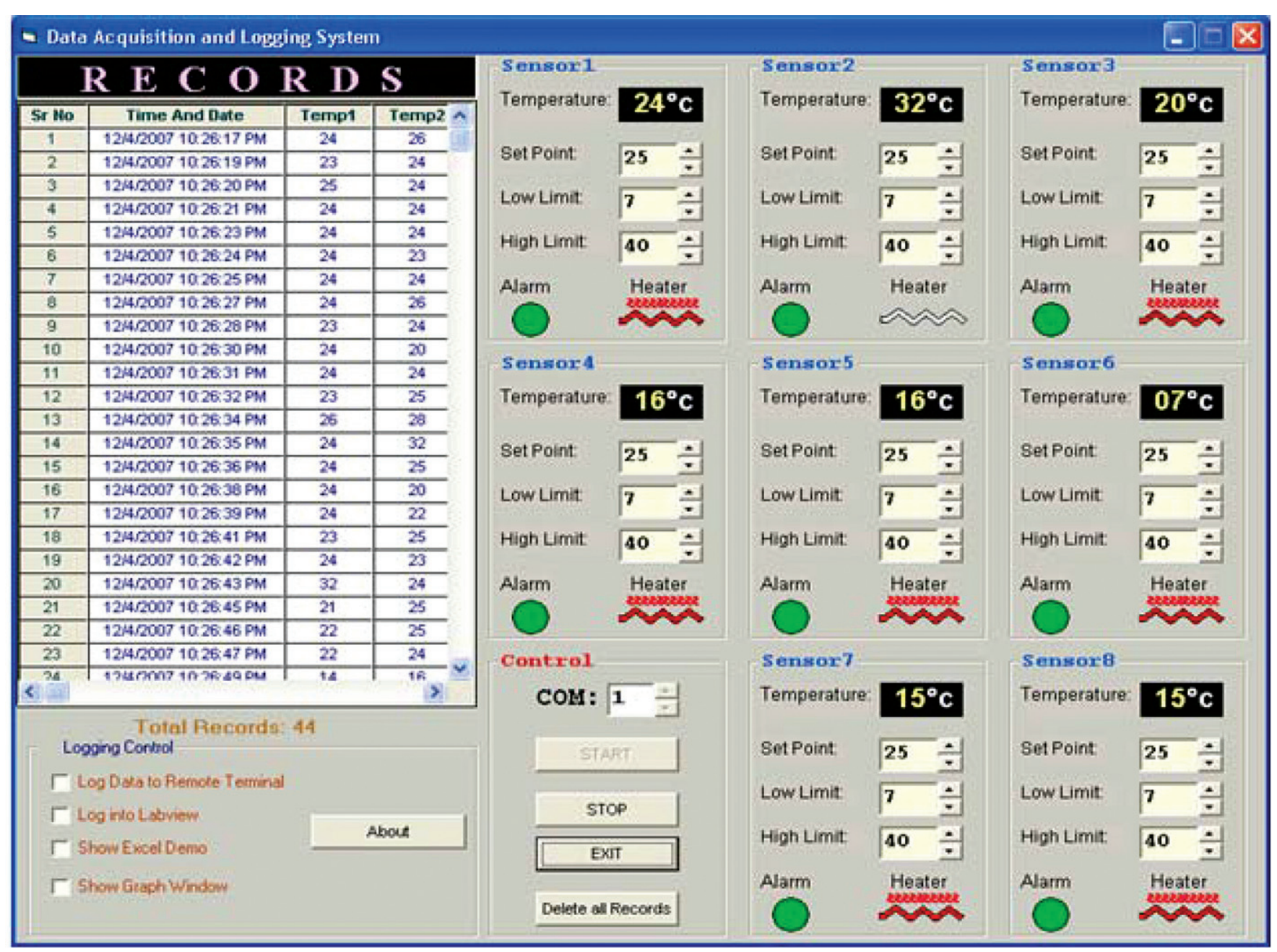

Fig.4

plant to adjust their operation, i.e., to improve the MPPT operation.

In 2016, a solar power project commissioned the world's largest solar plant in a single location, a strong contribution to India's goal of $100 \mathrm{GW}$ of solar power by 2020. Built in Kamuthi, $90 \mathrm{~km}$ from Madurai, the solar project surpasses the California-based Topaz Solar farm which has a capacity of 550 MW.

The massive 648-MW solar project, with five separate plants, contributes to the country's renewable energy vision, with the overall aim of diversifying the energy mix to meet growing demand, while minimizing the environmental impact. The plant, spread over 2,500 acres (10 sq km), enables clean power supply for around 150,000 households, based on an average national per capita consumption, and accounts for about 10 per cent of India's solar power output.

Automation solutions were roped in for end-to-end power and automation solutions to the solar power plant. (Fig.4).
All equipment across the plant is integrated and controlled with the industry proven plus SCADA system a robust automation platform, forming the digital backbone of the massive solar power plant. Equipment across the plant, including inverters, switchyard, switchgears and weather stations, are monitored and controlled using sophisticated software from a common automation system.

Due to its large scale, 648 (MWh), any fluctuation in the plant output power will affect the grid operations. The project works on a first-of-its-kind control philosophy to maintain the power output of the plant at the grid demand.

The control philosophy also tracks the change in weather conditions like cloud cover, which immediately affects the power generation. The control system adjusts the inverter setpoint and maintains steady output power.

There are five automation systems - one for each substation and a central control room. This automation system offers a single view of the plant, enabling plant performance monitoring, enhanced operational efficiency and ensuring grid compliance. IoT based automation system 
facilitates local and remote monitoring and control of the plant and substation assets. Plant wide SCADA solutions ensure that all relevant plant data is stored and analyzed by diagnostics applications to improve efficiency of the plant.

Remote monitoring solutions further improve the effectiveness of operations and maintenance of solar plants, boosting capabilities for remote control, diagnostics and predictive maintenance. Overall plant control schemes ensure grid code compliance, around the clock, around the globe.

The remote service platform contains a set of tools to detect and correct the most common reasons for underperforming assets. The tools analyze the plant in small sections (typically individual strings) to pinpoint local problems at an early stage before they develop into larger production problems. They detect soiling (dust accumulation on the modules); total and partial shading of strings; and aging, which analyzes the efficiency of the PV modules over time to determine the loss in performance caused by degradation.

\subsection{Conclusion}

Internet ready automation systems with cyber security features enable solar power plants to operate with the demands following the grid code and ease of maintenance coupled with energy auditing features.

\section{References}

[1] Li, D., Serizawa, Y. and Kiuchi, M.: Concept Design for a Web-based Supervisory Control and DataAcquisition (SCADA) System, in Proc. IEEE PES Transmission and Distribution Conference and Exhibition.

[2] Goel, A. and Mishra, R.S.: Remote Data Acquisition Using Wireless-SCADA System, International Journal of Engineering (IJE), Vol. 3, Issue 1,

[3] www.wikipedia.com.

[4] IEEE Std C37.1-2007 (revision of IEEE Std C37.1-1994), IEEE standard for SCADA and automation systems.

[5] Kearney. D. (1989): Solar Electric Generating Stations (SEGS), IEEE Power Engineering Review, vol. 9, no. 8, pp. 4-8, doi:10.1109/MPER,4310850.

[6] Camacho, E.F., Berenguel, M., Rubio, F.R. and Martinez, D. (): Control of Solar Energy Systems. Berlin: Springer Verlag.

[7] National Academy of Engineering. Grand Challenges for Engineering [Online], 2008.

[8] Koutroulis, E. and Kalaitzakis, K.: "Development of an integrated data-acquisition system for renewable energy sources systems monitoring."

\section{STATEMENT OF OWNERSHIP AND OTHER PARTICULARS ABOUT INDIAN JOURNAL OF POWER \& RIVER VALLEY DEVELOPMENT REGISTRATION OF NEWSPAPERS (CENTRAL RULES, 1956 (AS AMENDED) FORM IV (Rule 8)}

1. Place of publication

2. Periodicity of its publication

3. Printer's Name

Whether citizen of India ? Address

4. Publisher's Name Whether citizen of India ? Address

5. Hony. Chief Editor Whether citizen of India ? Address

6. Managing Editor Whether citizen of India ? Address

7. Names and addresses of individuals who own the newspaper and partners and shareholders holding more than one per cent of the total capital.

I, Pradip Kumar Chanda hereby declare that the particulars given above are true to the best of my knowledge and belief.
... 62 Lenin Sarani, Kolkata 700013

... Bi-monthly

... Pradip Kumar Chanda

... Indian Citizen

... 62 Lenin Sarani, Kolkata 700013

... Pradip Kumar Chanda

... Indian Citizen

... 62 Lenin Sarani, Kolkata 700013

... Jayanta Bhattacharya

... Indian Citizen

... 62 Lenin Sarani, Kolkata 700013

... Pradip Kumar Chanda

... Indian Citizen

... 9A Sarat Chatterjee Road, P.O. Nabagram 712246, Hooghly BOOKS \& JOURNALS PRIVATE LTD.

Shareholders :

Pradip Kumar Chanda, 9A Sarat Chatterjee Road, P.O. Nabagram 712246, Hooghly Mrs. B. Chanda, 9A Sarat Chatterjee Road, P.O. Nabagram 712246, Hooghly.

\section{$\mathrm{Sd} /$ \\ Pradip Kumar Chanda Publisher}

Date : 28th April 2021 